\title{
LA CÁTEDRA DE ASTROLOGÍA Y MATEMÁTICAS EN LA REAL Y PONTIFICIA UNIVERSIDAD DE MÉXICO
}

\section{Martha Eugenia Rodríguez}

En el siglo XVII se hizo necesario el estudio de la astrología y de las matemáticas entre los médicos que habitaban la Nueva España, por lo que se abrió una cátedra en la carrera de medicina que se impartía en la Real y Pontificia Universidad de México. Su apertura se debió a la solicitud de fray Diego Rodríguez, quien fue bachiller en Artes y en Teología, títulos que obtuvo en la Universidad mencionada. Asimismo fue comendador en el convento de la orden de Nuestra Señora de la Merced.

Cabe recordar que la Facultad de Medicina se inauguró en 1578 con la cátedra de Prima de Medicina. En el mismo siglo XVI se creó otra asignatura más, la de Vísperas de Medicina y en el siguiente siglo se conformó también con las de Metodo Medendi, Anatomía y Cirugía y por último con la de Astrología y Matemáticas.

Antes de comentar cómo se creó la cátedra de Astrología y Matemáticas y su contenido, es conveniente mencionar que el estudiante que aspiraba a estudiar medicina ya tenía una base científica en virtud de que era requisito cursar las materias del trivium y del cuadrivium; es decir, la gramática, la retórica y la dialéctica dentro del trivium y la aritmética, 
geometría, astrología y física por otra parte, enmarcadas dentro del cuadrivium.

El 22 de febrero de 1637, en claustro pleno de la Universidad, se reunieron los conciliarios con el objeto de estudiar lo referente a una nueva cátedra, la de Astrología y Matemáticas. A través del documento que resultó de aquella sesión se puede apreciar que dicha materia se creó por iniciativa de su primer profesor, fray Diego Rodríguez, pero también por petición de los alumnos. El acta de la sesión dice:

«... habiendo visto lo pedido por el Padre presentado Fray Diego Rodríguez... y el ofrecimiento que hace a esta dicha Universidad de leer en ella la cátedra de Matemática, y así mismo lo pedido por los cursantes de la Facultad de Medicina, en esta razón y ser como es la dicha Cátedra de tanta utilidad y provecho para los dichos cursantes y Universidad, dijeron que aceptaban y aceptaron el dicho ofrecimiento en nombre de la dicha Universidad...» (1).

En la orden de creación que daba el virrey de la Nueva España, el marqués de Cadereita afirmaba respecto a fray Diego Rodríguez que

«... ha más de treinta años que está estudiando las ciencias matemáticas con notable solicitud y cuidado, de que en todas las ocasiones que se han ofrecido ha dado suficiente muestra de sus estudios, y hecho diversos escritos y tratados de las dichas ciencias, por lo cual la Real Universidad de la Ciudad, en su Claustro le nombraron y dieron licencia para que en ella lea públicamente la Cátedra de Matemática...».

Y. efectivamente fray Diego Rodríguez era un serio estudioso de las matemáticas. Fue autor de diversos escritos, entre ellos Geometría especulativa, De la naturaleza, generación y propiedades de los números cubos y sus compuestos, hasta hoy nunca tratados por autor alguno, De aritmética, Tratado de ecuaciones, con tratado algebraica discursiva, su uso y su formación y por último Discurso etheorológico sobre el cometa aparecido en México en 1652. Asimismo fray Diego Rodríguez fue un académico interesado en ampliar sus conocimientos y en establecer correspondencia con sus colegas europeos. 
Fray Diego Rodríguez pudo empezar a leer la cátedra de Astrología y Matemáticas bajo ciertas condiciones: tenía precisa obligación de asistir a todas las lecturas de la materia y no ausentarse sin licencia; la lección se impartiría de 10 a 11 de la mañana; su nombramiento como profesor no tendría limitación de tiẹmpo, y testimonio de esto es que Rodríguez permaneció en el cargo hasta el momento de su muerte, en 1668. Se le señalaban 100 pesos de salario por cada año. Asimismo, la aprobación concedida por el claustro respecto a la nueva cátedra debía ser ratificada por el virrey marqués de Cadereita, en su calidad de patrón de la Universidad y como representante de su majestad. La autorización del virrey fue fechada el 23 de marzo de 1637 y 3 días más tarde el catedrático tomó posesión de su cargo.

A lo largo del periodo virreinal esta cátedra contó con muchos maestros sobresalientes. Además del propio fray Diego Rodríguez, figuraron también don Carlos de Sigüenza y Góngora, Joaquín Velázquez de León y José Ignacio Bartolache.

La creación de esta cátedra en la Nueva España fue resultado de las teorías dominantes en el viejo mundo donde se creía que la posición de los astros influía sobre los fenómenos fisiológicos.

A diferencia de las otras asignaturas que integraban la carrera de medicina, la de Astrología y Matemáticas fue impartida en lengua romance. Se dictaba en castellano con el objeto de que fuera de provecho no sólo para los médicos sino también para los que quisieran ser arquitectos, agrimensores o calendaristas y cuyas carreras no exigieran saber la lengua latina. Estos cuatro profesionales debían cursar la materia para saber la mecánica, la hidráulica, óptica, aerometría, aritmética y geometría. Sin embargo, para los estudiantes de medicina la materia cobró el carácter de obligatoria.

Un hecho que causó mucha controversia en el virreinato fue el decidir a quien correspondía impartir la cátedra. En la Recopilación de leyes de los Reynos de las Indias (2) publicadas por el rey don Carlos II en 1681, se mencionaba que la cátedra de Matemáticas la debía enseñar un cosmógrafo. Para el siguiente siglo, el 3 de julio de 1757 se dio una real cédula en Aranjuez mandando que a partir de esa fecha el profesor de la cátedra de Astrología y Matemáticas tendría que tener el título de doctor en medicina, es decir, el grado más alto que otorgaba la Universidad, pues cabe recordar que los estudiantes de medicina podían obtener los títulos de bachiller, licenciado o doctor. El título de maestría sí lo otorgaba la Universidad pero en otras carreras. Sin embargo, en marzo de 1773, a peti- 
ción del doctor José Ignacio Bartolache, el claustro universitario declaró que las matemáticas debían ser impartidas por una persona especializada y no simplemente por cualquier médico, en virtud de que era materia fundamental de muchas disciplinas.

La cátedra de Astrología y Matemáticas no figuró en los estatutos universitarios elaborados por don Juan de Palafox y Mendoza en 1688, pero sobre la base de las constituciones de la Universidad de Salamanca se puede conocer su plan de estudios. Dicha asignatura comprendía temas como la condensación y rarificación de los cuerpos, los cuerpos tensocompresos y extensos y su fuerza elástica, los elementos botánicos, la física mecánica, la geometría, astronomía, cosmografía, matemáticas, farmacología, química y geografía (3).

Sobre las lecturas se ordenaba en la Recopilación de leyes de los Reynos de las Indias lo siguiente:

Para el primer año se leería la Esfera de Juan Sacrobosco, las cuatro reglas de aritmética, regla de tres, raíz cuadrada y cúbica, algunas reglas de quebrados; las teorías acerca del sol de Purbaquio y las tablas astronómicas del señor rey Alfonso el Sabio.

Para el segundo año se leerían los seis primeros libros de los Elementos de geometría de Euclides; los arcos y cuerdas, senos rectos, tangentes y secantes; el libro cuarto de los Triángulos esferales de Juan de Monteregio y el Almagesto de Ptolomeo.

En el tercer año se debía leer sobre cosmografía y navegación, sobre el uso del astrolabio y del planisferio, sobre cómo hacer observaciones de los movimientos del sol, la luna y los planetas; el uso del radioglobo y algunos otros instrumentos matemáticos.

Por las constituciones salmantinas se sabe que también eran leídas la Sphaerica de Theodosio y los libros de Copérnico titulados Sobre las revoluciones de los cuerpos celestes.

Por el programa de lecturas se puede ver que el estudiante de la asignatura que comentamos adquiría una amplia visión del tema, haciendo un estudio diacrónico de los científicos, partiendo de la antigüedad, donde destaca Euclides, cuyos principios sirvieron de base a la geometría moderna, pasando posteriormente por Ptolomeo, con quien culminó la astronomía, la matemática y la geografía de la antigüedad, hasta llegar a los autores que realmente tenían un espíritu científico, como Copérnico, iniciador de la cosmografía científica en sentido riguroso.

Por si las lecturas anteriores no eran suficientes, las Leyes de los Reynos de las Indias decían: 
«En los meses de vacaciones podrá leer materias de reloxes, y mecánicas, con algunas máquinas, y dar á entender en que consiste la fuerza dellas..."

Otros libros que podían servir de complemento para los cursantes de medicina eran la Verdadera medicina, cirugía y astrología del doctor Juan de Barrios, El sitio y naturaleza de la ciudad de México del doctor Diego Cisneros y el Repertorio de los tiempos de Enrico Martínez, todos escritos en la Nueva España en el siglo XVII. En el siguiente siglo Bartolache escribió sus Lécciones de matemáticas.

Con el correr del tiempo, en ocasiones la cátedra de Astrología y Matemáticas fue considerada como materia exclusiva para los estudiantes de medicina y otras veces se vio como cultura general, contando entonces con una mayor asistencia.

La creación de la cátedra de Astrología y Matemáticas en la Universidad de México obedeció a que desde la antigüedad clásica se desarrolló la idea de relacionar el universo o macrocosmos con el cuerpo humano o microcosmos. Parece ser que el término microcosmos procede de Demócrito, quien habla de que el hombre es un mundo en pequeño. Esto lo ratificaban al ver que cuando había pérdida de la cosecha por el mal tiempo, como consecuencia también había hambre y pestes, es decir, existía una relación de causa-efecto. El hombre, entendido como el microcosmos, era gobernado por el macrocosmos, es decir, por los movimientos de los planetas y la disposición de las estrellas en el universo. De acuerdo con esta relación de macrocosmos y microcosmos, los médicos hablaban de una naturaleza del universo y una naturaleza humana, donde había una proporcionalidad de elementos. La composición del cuerpo humano constaba, de acuerdo con la mentalidad de la época, de cuatro cualidades correspondientes a los cuatro elementos, es decir, aire, fuego, agua y tierra. Dichas cualidades se denominaban humores y su equilibrio daba lugar a la salud del individuo. Los cuatro humores que integraban el cuerpo humano eran la sangre, la bilis amarilla o cólera, la flema o pituita y la melancolía o bilis negra.

En la Nueva España del siglo XVII se creía que había en algunos hombres ciertas propiedades e inclinaciones que no podían provenir sólo del temperamento del cuerpo; algunas propiedades no dependían sólo del frío, calor, humedad o sequedad, que eran cualidades particulares de los humores, sino de una oculta influencia celeste, es decir, había una estre- 
cha relación entre la astrología y la medicina, debido a la vigencia que seguían teniendo las obras de la antigüedad. La concepción microcósmica del hombre adquirió en la Grecia clásica una significación cósmico-fisiológica, más que cósmico-religiosa.

Un escrito del Corpus hippocraticum en el que se pone de manifiesto el pensamiento microcósmico es el que se titula Sobre la dieta. Aquí el cuerpo del hombre es "copia del todo", imitación del universo. Se decía que el firmamento correspondía a la piel, el mar al vientre y la tierra al estómago y al pulmón, es decir, la doctrina del microcosmos se fisiologizó. "Lo esencial del paralelismo entre el mundo y el hombre estaría en los circuitos de sus respectivos movimientos diarios, semanales, mensuales y anuales; en sus respectivos "ritmos», como ahora es costumbre decir. Ritmos a cuya estructura pertenecería, tanto en el mundo como en el hombre, una armonía susceptible de reducción a proporción numérica» (4).

Lo que sucede en el cosmos sirve para entender lo que acontece en el hombre y viceversa. Hay una correlación entre los períodos del año, las estaciones, la dinámica de los humores y la producción de las enfermedades. Por tanto, cabe concluir que el pensamiento médico de la antigüedad clásica estaba traspasado por la concepción microcósmica de la naturaleza humana y estos mismos conceptos son los que se enseñaban en la universidad novohispana, agregando, claro está, dentro de la cátedra de Astrología y Matemáticas las teorías modernas de la cosmografía.

La medicina, desde sus más remotos tiempos, ha basado sus conocimientos y experiencias en el aumento y disminución del dolor, en el ciclo mayor y menor de las horas y en el ritmo del tiempo a fin de percatarse de los momentos clave en las crisis.

Dadó que la enseñanza que se impartía en la Universidad novohispana estaba basada en los autores de la antigüedad clásica y medievales, es válido recurrir a ellos para saber en qué consistía el contenido de las materias. Para la de Astrología y Matemáticas, Casiodoro, en sus Instituciones, decía:

«la aritmética es una enseñanza tan grandiosa como provechosa para nuestra vida, pues mediante su ayuda nos percatamos de la existencia de nuestro propio yo, permitiéndonos además calcular la medida de lo pasado al ser capaces de calcular de un modo equilibrado" (5). . 
Lo anterior se aplicaba tanto a los días sanos como a los de enfermedad.

La aritmética era algo más que el simple arte de calcular. Tenía mucho que ver con el ritmo de nuestra existencia. San Isidoro sostenía que el médico tenía que aprender aritmética para contar las horas en que se desarrolla una enfermedad y para conocer el ritmo de los días decretorios.

Los días decretorios o días judicatorios o de crisis se llamaban así porque era cuando se juzgaba si la enfermedad declinaría a bien o a mal. En esos días de crisis había una lucha entre la virtud natural del enfermo y el humor causador de la enfermedad. Enrico Martínez (6), famoso cosmógrafo de la Nueva España, definía a la enfermedad siguiendo la tradición hipocrática, como la descomposición o desequilibrio de alguno de los humores.

Por su parte, la geometría fue entendida como ciencia de la medida. Mide y describe la tierra, observa las correlaciones del clima y la influencia que tiene cada grado de altura. El médico debía valerse de la geometría con el objeto de llegar a conocer las peculiaridades cualitativas de las diferentes partes de la tierra y la situación de cada región. De igual manera la medicina era una disciplina del ambiente, ya que éste influía en el organismo sano y en el enfermo.

El pensador latino Boecio, en su tratado titulado Sobre la geometría, sostiene que esta disciplina tiene diversas utilidades, como por ejemplo, en la arquitectura. Al médico le era necesaria para la observación del cuerpo en los días sanos y en los de enfermedad. A través de la geometría el médico podía llegar a compenetrarse cada vez más con el orden cósmico.

En el siglo XII el médico Pedro Alfonso sostenía en su escrito denominado Carta sobre el estudio que la aritmética permitía a la medicina escoger los principios, con sus nexos, clases y estadios, los pesos de los medicamentos y determinar las enfermedades, los días y semanas, los grados de fiebre y otras cosas de importancia.

Además de la aritmética y de la geometría, la medicina sólo podría ser dominada con ayuda de la astronomía. Si el médico tenia conocimientos astronómicos podía determinar más fácilmente una terapia que evitara las enfermedades o que las curara de manera más rápida. Asimismo podría regular la toma de medicamentos y establecer el día y hora en el ciclo de la fiebre.

Enrico Martínez diferenciaba bien la Astronomía de la Astrología, ambas de mucha utilidad para el médico. Recurrimos a Martínez porque, 
aunque no fue oriundo de la Nueva España, sí se desenvolvió como científico en estas tierras, y precisamente en el siglo XVII, cuando se creó la cátedra de Astrología y matemáticas en la Universidad.

Dicho autor sostenía que la astronomía «trata de los movimientos de los cielos y planetas, de sus varias conjunciones, oposiciones y concursos»; en cambio la astrología «enseña a saber los efectos que los movimientos, conjunciones y aspectos de los cuerpos celestes causan en estas cosas inferiores» (7), pues era generalizada la idea de que el organismo humano cambiaba de acuerdo con la posición de las estrellas. Nuestra salud dependía en parte de las leyes del universo. El médico estaba muy relacionado con el firmamento o sistema natural por lo que se le llamó physicus o filósofo de la naturaleza. Esto se explica porque el ser humano, como ya se comentó, es en todo igual al mundo espacial, a las cosas del universo, como por ejemplo, los vasos sanguíneos se asemejaban a los ríos.

La astrología era necesaria al médico dado que la influencia celeste causaba efectos en el cuerpo humano. Los humores que integraban el organismo se descomponían por dos causas: por los excesos que cometía el hombre y por la poca precaución que tenía, como por ejemplo, un abuso en los alimentos, y por influencias celestes, pues la posición de los astros tenía una acción sobre la fisiología humana. Por ejemplo, los enfermos de bubas y los que padecían dolores en las junturas del cuerpo recibían alivio con la luz del día y con la presencia del sol, mientras que con su ausencia se agravaba el mal. El sol recreaba a la naturaleza humana.

Martínez sostenía que era deber del astrólogo dar consejos al médico para ayudarlo en su profesión. Tenía que saber que no todos los días eran apropiados para aplicar remedios como las purgas y sangrías, algunos eran peligrosos.

En el tratado de Galeno que lleva el título de Días críticos se habla de la influencia de la luna sobre los seres humanos. Estas mismas ideas seguían vigentes en la Nueva España. Ejemplo de ello es que Enrico Martínez sostenía que el origen y cuenta de los días decretorios procede del curso y movimiento de la luna, la cual, según Galeno, en el tercero de los días decretorios causa efectos muy evidentes en estas cosas inferiores, en especial en los cuerpos humanos; y no sólo sienten sus operaciones los enfermos, mas también los sanos, ya que el hombre es «un mundo abreviado que participa de todo y conviene que la parte siga al todo como el efecto a su causa». Si una cierta calidad e influencia celeste no hacía igual efecto en todos los hombres, era por la diversidad de las complexiones de ellos. 
Enrico Martínez sostenía que no era supersticioso aprovecharse de la astrología en la medicina, sino útil y necesario. Esto se comprobaba por la constitución que el papa Sixto V había dado en Roma el 5 de enero de 1586, en la cual prohibía todas las sectas judiciarias, excepto la astrología que trata, según afirma, acerca de la agricultura, la navegación y sobre cosas de medicina. Es decir, esto prueba que era aceptado que la medicina recurriera a la astrología.

Dadas las causas de las enfermedades, el paciente contaba con dos vías para remediar su mal: una por medio de la influencia celeste y la segunda por medio del arte de la medicina. Cuando se unían estas opciones, ambas con la ayuda de las matemáticas, solían hacer aventajado efecto.

La cátedra de Astrología y Matemáticas fue de gran trascendencia en la evolución de la educación universitaria de la Nueva España debido a que dio a conocer los últimos avances del pensamiento científico europeo, como por ejernplo la obra de Copérnico.

En principio, el título de la cátedra que venimos comentando podría hacer pensar que se trataba de una materia de carácter supersticioso, pero si nos trasladamos a la mentalidad de la época, se ve que no fue así.

La Facultad de Medicina fue el recinto donde se manifestó el interés por adoptar las ideas modernas del viejo mundo, y la cátedra de Astrología y Matemáticas marcó una diferencia con las otras asignaturas del programa académico de medicina. Estableció la transición a la renovación de los estudios universitarios, en virtud de que contaba con lecturas de autores renacentistas, además de los de la Antigüedad y de la Edad Media, claro está. En las otras materias del plan de estudios no se leían a autores modernos, eran totalmente tradicionalistas.

La relación astrología-medicina parece más común que la relación matemáticas-medicina, pues suele vincularse, aún hoy en día, con la práctica de la medicina popular. Sin embargo, en la historia de la medicina universal, la astrología ha tenido mucho que ver con la denominada medicina científica, cuyo origen data de la Grecia antigua, y precisamente por el vínculo que existía entre la medicina y la astrología, era necesario recurrir al estudio de las matemáticas. Recuérdese que inicialmente la cátedra la tenía que impartir un cosmógrafo, quien cubría los dos aspectos de la materia.

Las matemáticas fueron indispensables para conocer la estructura del universo y para hacer cálculos astrológicos, Aunque hoy en día parezca curioso y absurdo, profesar la astrología era cosa común y respetada, al grado que se enseñaba en la universidad. En la época que nos ocupa hu- 
bo una matematización del saber. Conocer el cosmos era medirlo y matematizarlo, ya que el universo estaba constituido por cuerpos materiales variables en su forma y contenido y, para entender esa apariencia, era preciso concebirla reduciéndola metódicamente a una combinación de figuras geométricas, figuras de las que la mente humana podía dar razón. Por tanto, las matemáticas le sirvieron al médico para conocer el cosmos y, desde luego, para apreciar mejor el estado clínico del paciente, los días decretorios y el ritmo de las fiebres.

La creación de la cátedra de Astrología y Matemáticas en la universidad novohispana obedeció a la regla que aquí existía de adoptar las ideas y costumbres de la Metrópoli, en este caso los planes de estudio, que por años se venían enseñando en las universidades españolas, donde estaba arraigada la relación de la medicina con las matemáticas y la astrología.

La cátedra que hemos comentado se enseñó prácticamente durante doscientos años; llegó a su fin en 1833, fecha en que el poder ejecutivo hizo modificaciones de la enseñanza pública en todos sus ramos. Cerró la Facultad de Medicina y en su lugar creó el Establecimiento de Ciencias Médicas, el cual contó con un nuevo plan de estudios, actualizado a las necesidades de la época.

\section{BIBLIOGRAFÍA}

(1) Francisco Fernández del CASTillo (1953). La Facultad de Medicina según el archivo de la Real y Pontificia Universidad de México, México, Consejo de Humanidades UNAM, 314 p., ils.

(2) Recopilación de leyes de los Reynos de las Indias mandadas imprimir y publicar por la magestad católica del Rey Don Carlos II Nuestro Señor (1861), Madrid, 1791, folio 186.

(3) Estatutos hechos por la Universidad de Salamanca, recopilados nuevamente por su comisión. Impresos en Salamanca por Diego Cufio el año de 1625. Archivo General de la Nación, Ramo: Universidad, tomo 4, volumen 249, folio 183

(4) Pedro Laín Entralgo (1982). La medicina hipocrática, Madrid, Alianza Editorial $456 \mathrm{p}$.

(5) Heinrich SCHIPPERGER (1982). "La medicina en la Edad Media latina» en Historia Universal de la Medicina, dirigida por Pedro Laín Entralgo, tomo III, Barcelona, Salvat Editores, 354 p., ils.

(6) Henrico Martínez (1948). Reportorio de los tiempos e historia natural de Nueva España (1606), Introducción Francisco de la Maza, México, Secretaría de Educación Pública, 318 p., (Testimonios Mexicanos, Historiadores 1).

(7) Idem p. XVII. 